\title{
On the relationship between response selection and response inhibition: An individual differences approach
}

\author{
Angela D. Bender ${ }^{1}$ • Hannah L. Filmer ${ }^{1}$ • K. G. Garner ${ }^{1}$ - Claire K. Naughtin ${ }^{1}$. \\ Paul E. Dux ${ }^{1}$
}

Published online: 5 July 2016

(C) The Psychonomic Society, Inc. 2016

\begin{abstract}
The abilities to select appropriate responses and suppress unwanted actions are key executive functions that enable flexible and goal-directed behavior. However, to date it has been unclear whether these two cognitive operations tap a common action control resource or reflect two distinct processes. In the present study, we used an individual differences approach to examine the underlying relationships across seven paradigms that varied in their response selection and response inhibition requirements: stop-signal, go-no-go, Stroop, flanker, single-response selection, psychological refractory period, and attentional blink tasks. A confirmatory factor analysis suggested that response inhibition and response selection are separable, with stop-signal and go-no-go task performance being related to response inhibition, and performance in the psychological refractory period, Stroop, single-response selection, and attentional blink tasks being related to response selection. These findings provide evidence in support of the hypothesis that response selection and response inhibition reflect two distinct cognitive operations.
\end{abstract}

Keywords Executive control $\cdot$ Response selection $\cdot$ Response inhibition $\cdot$ Individual differences

The abilities to select responses to specific stimuli (i.e., response selection) and to override a prepotent tendency to

Electronic supplementary material The online version of this article (doi:10.3758/s13414-016-1158-8) contains supplementary material, which is available to authorized users.

Angela D. Bender

a bender1@yahoo.com.au

1 School of Psychology, University of Queensland, McElwain Building, St Lucia, QLD 4072, Australia respond to other stimuli (i.e., response inhibition) have been attributed to core executive action control processes (Norman $\&$ Shallice, 1986). These functions are critical in everyday life, because they allow people to flexibly adjust their behavior according to their goals. Indeed, deficits in these cognitive operations contribute to several psychopathological conditions, including obsessive-compulsive disorder (Penades et al., 2007), attention-deficit hyperactivity disorder (Nigg, 2001), and addiction and eating disorders (Crews \& Boettiger, 2009; Houben, 2011; Noel, Brevers, \& Bechara, 2013). However, despite the similarities between response selection and inhibition - that is, both require the selection or nonselection of a response - the extent to which these processes relate and interact with each other is still unknown.

Response selection is a central decision-making process that maps incoming perceptual information to goal-appropriate motor output (Pashler, 1984; Welford, 1952). One theoretical framework that explicitly illustrates the various steps involved in the decision-making process is sequential-sampling models. According to these models, simple decision-making involves the accumulation of information from the environment until a certain response criterion threshold (i.e., the amount of information needed to select a response) has been reached (Brown \& Heathcote, 2008; Ratcliff \& Smith, 2004; Smith \& Ratcliff, 2004; Usher \& McClelland, 2001). The option that reaches the response criterion threshold first is selected and then executed. Whereas the processes at the perceptual and motor stages are thought to operate in parallel, the central response selection stage has been proposed to be capacity-limited - only capable of acting on a single task at any given time. This sensory-motor translation stage is therefore often studied using paradigms such as the psychological refractory period (PRP; Pashler, 1984; Welford, 1952), dual-task (Dux, Ivanoff, Asplund, \& Marois, 2006; Dux et al., 2009; Schumacher et al., 2001; Sigman \& Dehaene, 2008), and high-response-load single-task tests, 
because these measures place strong demands on the response selection system. Although conditions differ across these paradigms, they all require participants to encode sensory information and make a decision, and in paradigms with two tasks, participants must make simple response selections for items that occur simultaneously or in close succession.

In contrast, response inhibition is thought to be a heterogeneous construct that can be parsed into at least two different forms: task-relevant and task-irrelevant inhibition (Harnishfeger, 1995; Nigg, 2000). Successful inhibition of distractor stimuli is often measured in tasks where taskirrelevant information needs to be ignored, such as the Eriksen flanker task (Eriksen \& Schultz, 1979) and Stroop task (Stroop, 1935). Task-relevant response inhibition, however, is often measured in tasks such as the stop-signal (Lappin \& Eriksen, 1966; Verbruggen \& Logan, 2008) and go-no-go (Donders, 1969) paradigms, which require the inhibition of task-relevant, prepotent response tendencies on a subset of trials. According to the popular race model account (Logan \& Cowan, 1984), successful inhibition in the stop-signal task relies on the outcome of a race between independent "go" and "stop" processes. Inhibitory control succeeds when the stop process finishes the race before the go process, whereas inhibition fails if the go process reaches the response threshold first.

The attentional blink $(\mathrm{AB})$ is another dual-task paradigm worth noting, since it is thought to tap both response selection and inhibition operations. The $\mathrm{AB}$ is a perceptual limitation that is reflected in the inability to consciously perceive and report the second of two targets presented in close succession (Jolicœur \& Dell'Acqua, 1998; Raymond, Shapiro, \& Arnell, 1992). Although most explanations of the AB invoke a consolidation/response-selection bottleneck or cognitivecontrol mechanism(s) (Dux \& Marois, 2009), research suggests that a suppressive mechanism that inhibits the deployment of attentional resources to irrelevant distractor stimuli may also play an important role in target selection (Dux \& Harris, 2007; Dux \& Marois, 2008; Raymond et al., 1992; Wyble, Bowman, \& Nieuwenstein, 2009).

Traditionally, response selection and response inhibition processes have been investigated separately, but increasing evidence is suggesting that these two operations may draw on a common resource (Mostofsky \& Simmonds, 2008; van Gaal, Ridderinkhof, Fahrenfort, Scholte, \& Lamme, 2008; Verbruggen, McLaren, \& Chambers, 2014). For example, neuroimaging and transcranial magnetic stimulation (TMS) studies have shown that mid-dorsolateral and ventrolateral prefrontal areas (Bunge, 2004; Duncan \& Owen, 2000) and the presupplementary motor area (pre-SMA; Buch, Mars, Boorman, \& Rushworth, 2010) are activated by tasks that require the selection and inhibition of responses. For instance, Verbruggen, Aron, Stevens, and Chambers (2010) demonstrated that TMS of the right inferior frontal gyrus (IFG) impaired performance on both a stop-signal task and a dual task, suggesting that this region supports both response selection and inhibition processes. Taken together, these findings indicate that response inhibition and response selection protocols might tap, at least in part, a common mechanism of action control.

Although response selection and response inhibition processes may partially overlap at the neural level, evidence also suggests that response selection and response inhibition tasks recruit distinct brain regions. Indeed, neuroimaging studies have observed greater activity in the left hemisphere posterior lateral prefrontal cortex (pLPFC) during dual-task as compared to single-task trials, suggesting a role for this area in response selection (Dux et al., 2006; Dux et al., 2009; Jiang \& Kanwisher, 2003; Miller \& Cohen, 2001). Similarly, Filmer and colleagues demonstrated that transcranial direct current stimulation of the left but not the right pLPFC disrupts response selection and the associated training effects for both single and dual tasks (Filmer, Mattingley, \& Dux, 2013; Filmer, Mattingley, Marois, \& Dux, 2013). In contrast, right IFG appears to be crucial for the successful inhibition of an inappropriate motor response (Aron, Fletcher, Bullmore, Sahakian, \& Robbins, 2003), since it shows greater activity during successful as compared to failed inhibition trials (for a recent review, see Aron, Robbins, \& Poldrack, 2014). Moreover, TMS of the right IFG has been found to temporarily increase stop-signal response times (SSRTs) - an index of poorer inhibitory control-but TMS of other cortical regions, including the left IFG, right middle frontal gyrus, and right angular gyrus had no impact on SSRTs (Chambers et al., 2007; Chambers et al., 2006). These studies suggest processspecific functional differences and converge with the idea that response selection and response inhibition produce distinct neural signatures; namely, the left pLPFC is involved in response selection, whereas the right IFG is involved in response inhibition. Although no study has directly assessed whether these two processes can be fully dissociated in the brain, the neural findings do at least suggest that these two processes could reflect distinct forms of action control.

The independence of response selection and response inhibition processes is further suggested by behavioral studies. Whereas dual-task contexts typically result in performance decrements when central response selection processes overlap in time, performance on various inhibitory control tasks indicates that the go process can occur independently from the stop process (activated by the appearance of the stop signal; Logan \& Burkell, 1986; Verbruggen \& Logan, 2009; but see Verbruggen \& Logan, 2015). For example, a recent study by Yamaguchi, Logan, and Bissett (2012) directly examined stop-signal performance in a PRP task, in which participants were required to correctly inhibit one response while carrying out the other response in a dual-task setting. In line with the independence assumption, response inhibition performance was not influenced by dual-task interference, supporting the notion that response inhibition is distinct from response 
selection demands. In agreement with the aforementioned neural work, these findings further suggest that response selection and response inhibition operations reflect different elements of action control.

While the above-mentioned studies provide valuable insight into the cognitive and neural substrates of action control, they also highlight the lack of clarity about whether a general action control mechanism underpins performance in a wide range of response selection and inhibitory control paradigms, or whether the differences that we observe in these tasks reflect distinct underlying processes. The previous work has been unable to resolve this conflict because the studies have only compared single examples of response selection and inhibition tasks to test for possible overlap or dissociation between the two processes. A drawback of this approach is that it is impossible to infer the specific nature of the process (which is independent of the paradigm used) that leads to any observed overlap or dissociation. This problem can be overcome by employing a latent variable approach to better understand the relations among commonly used measures of response selection and response inhibition (Miyake et al., 2000). Confirmatory factor analysis (CFA) is a form of latent variable analysis that statistically extracts the common variance among tasks that are expected to tap the same putative function. By only analyzing what is common among the tasks, and excluding the systematic variance attributable to non-cognitivecontrol processes (e.g., sound and color processing), the "purer" latent variable (Miyake et al., 2000) can be extracted so as to examine how different cognitive-control functions relate to one another. Importantly, CFA allows the evaluation of different models on an a priori basis, utilizing knowledge from previous research about hypothesized task demands (i.e., tasks that are thought to share the same underlying construct should load on the same latent factor and are specified before the different models are run).

The application of CFA to exploring the relations among executive functions has been successfully demonstrated in a series of studies by Miyake and colleagues (Friedman \& Miyake, 2004; Miyake et al., 2000). Specifically, Miyake et al. (2000) used a battery of tasks thought to tap three core cognitive-control functions-shifting, updating the contents of working memory, and inhibition of automatic responses. Although the CFA results revealed that the three cognitive operations shared some common overlap, a three-factor model provided the best fit to the obtained data, suggesting that all three functions represent distinct cognitive constructs that are qualified by shared variance between the executive-control factors. The diversity of cognitive control is further supported by Friedman and Miyake's (2004) study that investigated the extent to which various measures of inhibitory control are related to one another and how these functions contribute to other cognitive-control-demanding tasks. The authors focused primarily on three potentially distinct inhibitory control functions: prepotent-response inhibition (the ability to override an automatic response), resistance to distractor interference (the ability to suppress irrelevant information), and resistance to proactive interference (the ability to suppress memory interference from previously relevant task information). CFA revealed that prepotent-response inhibition and resistance to distractor interference were closely related to each other, while resistance to proactive interference was unrelated to response and distractor inhibition.

Here, we employed a latent variable approach similar to that of Miyake et al. (2000) and Friedman and Miyake (2004), to understand the relations among response selection and response inhibition. This approach not only provides insights into how performance varies between individuals, but it can also identify the extent to which indicators of action control relate to each other. If response selection and response inhibition processes reflect two distinct aspects of action control, we would expect response selection tasks to load on one latent factor, and response inhibition tasks to load on a second factor. If, on the other hand, these two processes reflect "two sides of the same coin" (Mostofsky \& Simmonds, 2008), we would expect each paradigm to load highly on a single action control factor.

\section{Method}

\section{Participants}

Eighty-seven participants (mean age $=20$ years, range $=17-$ 39 years; 69 females, 18 males) from the University of Queensland participated in this study for course credit. All participants had normal or corrected-to-normal vision. The University of Queensland Human Research Ethics Committee approved the study, and all participants gave informed, written consent. The target sample and participant exclusion criteria were determined before data collection and were based on a 5:1 ratio of sample size to the number of free parameters (Bentler \& Chou, 1987). Participants were removed from the final analysis if they did not reach the behavioral cutoff for one or more tasks. In total, data from 21 participants were excluded from the final analysis, due to failure to attend a testing session (four participants) or because of poor performance (more than three standard deviations above the response time or accuracy mean) in one or several of the seven tasks (17 participants).

\section{Apparatus}

The experiment was run on an Apple Mac Mini computer and programmed in MATLAB (The MathWorks, Natick, MA) with the Psychophysics Toolbox extension (Brainard, 1997; Pelli, 1997). Stimuli were presented on a 21 -in. CRT monitor 
(100-Hz refresh rate), which participants viewed from a distance of approximately $57 \mathrm{~cm}$ during all the tasks in the two sessions.

\section{Procedure}

Participants took part in two 2-h sessions that were administered seven days apart from each other. During each session, participants completed the following tasks, with the task order randomized across participants and sessions: stop-signal task (SST), go-no-go task, Stroop task, flanker task, single- vs. dual-response selection paradigm, PRP, and AB. For all seven measures, each trial began with a black fixation cross that was presented in the center of a gray screen (RGB 128128 128) for a variable interval (200-600 ms). At the end of each block, the mean response time (RT) and accuracy were displayed (with the exception of the AB task, since participants are usually very poor at identifying the second target). Following the tasks in the first session, participants provided demographic information about their age, gender, handedness, years of education, history of neuropsychiatric illness, and current neuropsychiatric medication.

\section{Tasks}

\section{Response inhibition tasks}

Stop-signal task Participants completed go-signal and stopsignal trials. On go-signal trials, participants were required to discriminate between two different 2-D abstract shapes (gray with a black outline; see Fig. 1) by pressing the relevant response key ("F" or "J") as quickly and as accurately as possible. On stop trials ( $25 \%$ of trials), the primary go stimulus was followed by an auditory stop signal $(750-\mathrm{Hz}$ sine-wave tone, 200-ms duration), which instructed participants to inhibit their response. The stop-signal delay (SSD; i.e., the time between the onsets of the go stimulus and stop signal) was initially set at $250 \mathrm{~ms}$ and continuously adjusted with an adaptive staircase procedure to obtain a stopping probability of $50 \%$. If participants failed to inhibit their response in a stop-signal trial, the SSD decreased by $50 \mathrm{~ms}$, but if they succeeded, the SSD increased by $50 \mathrm{~ms}$. The fixation cross was replaced by the go stimulus, which remained on screen for $200 \mathrm{~ms}$. Participants completed one practice block of 24 trials and four test blocks of 36 trials. The dependent variable was the stopsignal RT (SSRT), calculated by subtracting each participant's mean SSD from the mean go RT (Verbruggen \& Logan, 2009). Better inhibitory performance was indicated by lower SSRTs.

Go-no-go task On each trial of this task, participants were instructed to make a speeded response to the go stimulus (a white, abstract 3-D shape; see Fig. 1 for an example) by
Fig. 1 Schematic representations of the paradigms and task performance on the seven measures of interest across Sessions 1 and 2. Task performance: (A) Mean go response times (Go-RT), the mean stopsignal delay (SSD), and the mean stop-signal RT (SSRT) in the stopsignal task. (B) Task performance in the go-no-go task: Mean go-RTs and commission errors (failed no-go trials). (C and D) Mean RTs for the congruent, incongruent, and neutral conditions in the Stroop and flanker tasks, respectively. (E) Mean RTs for the single and dual visual stimulus conditions and the single and dual auditory conditions in the single- vs. dual-response selection task. (F) Mean RTs as a function of stimulus onset asynchrony (SOA) for Target 1 (T1) and Target 2 (T2) in the psychological refractory period paradigm. (G) Mean accuracy as a function of lag for Target 2 accuracy, given that Target 1 is correct (T1 | $\mathrm{T} 2$ ), and $\mathrm{T} 1$ accuracy in the attentional blink task. The error bars represent the standard errors of the means (SEMs)

pressing the " $G$ " key, but to withhold from responding if the no-go stimulus appeared (another white, abstract 3-D shape; $25 \%$ of trials). The go and no-go stimuli were counterbalanced across participants. On each trial, a fixation cross appeared $(200 \mathrm{~ms})$, followed by one of the two target stimuli and an 1,800-ms response window. Participants completed a block of 24 practice trials and four test blocks of 36 trials. The dependent variable of interest was the number of commission errors on no-go trials (i.e., failure to inhibit a response). Better inhibitory control was indicated by fewer commission errors.

Stroop task In this task, participants had to report the presentation color (red, RGB 23732 36; green, RGB 10130 65; yellow, RGB 250250 0; or blue, RGB 4471 151) of both color ("red," "green," "yellow," and "blue") and noncolor ("cup," "fork," "spoon," "saucer") words as quickly and as accurately as possible via keypress. At the beginning of each trial, the fixation cross was replaced by a word target for $500 \mathrm{~ms}$. The word target was equally likely to be congruent (i.e., the printed color word matched its color on screen; e.g., "blue" printed in blue), incongruent (e.g., "blue" printed in red), or neutral (i.e., a noncolor word printed in any of the four colors; e.g., "cup" printed in green). Each of the four possible presentation colors was mapped onto a corresponding response key, and participants completed a practice block of 24 trials to familiarize themselves with the stimulus-response mappings. The experimental phase consisted of four blocks of 36 trials, and the order of trial types was randomized. The dependent variable was the "Stroop congruency effect," which was calculated as the difference in RTs between congruent and incongruent trials. A smaller congruency effect reflected better performance.

Flanker task Participants had to respond to the direction of a central arrow target $(>$ or $<$ ) as quickly and as accurately as possible, by pressing the " $<$ " key for leftward-pointing arrows and the ">" key for rightward-pointing arrows. On congruent trials, the target was flanked by two arrows on each side that 
a Stop Signal Task Resp window $1800 \mathrm{~ms}$ Go Trials

Fixation Target $200-600 \mathrm{~ms} 200 \mathrm{~ms}$

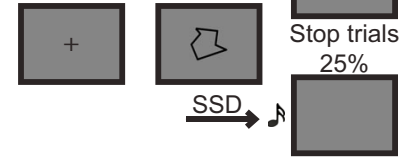

b Go No-Go Target Resp window
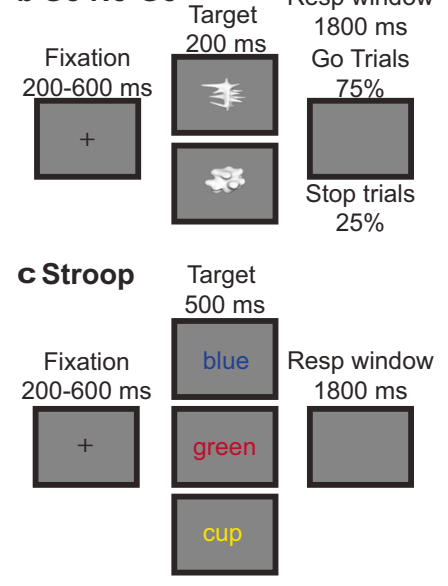

d Flanker Target

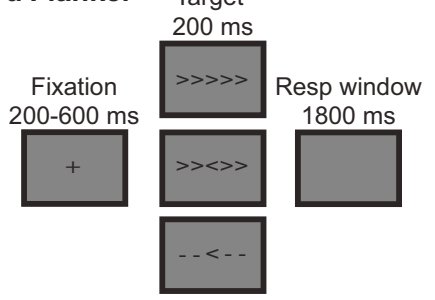

e dual-task Target

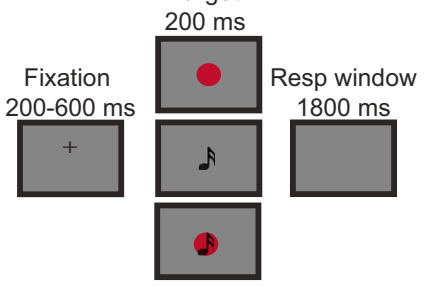

f PRP
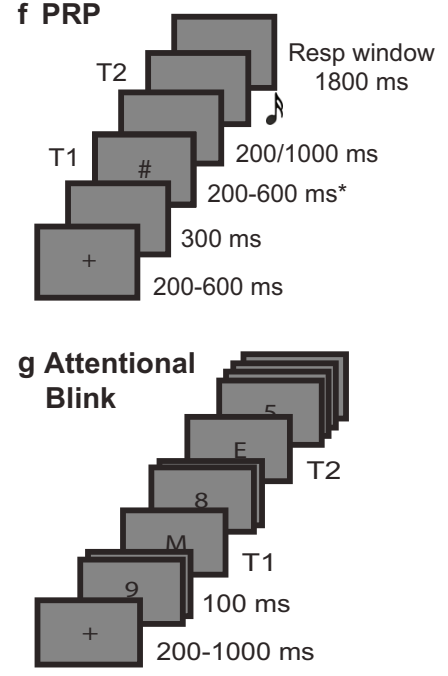
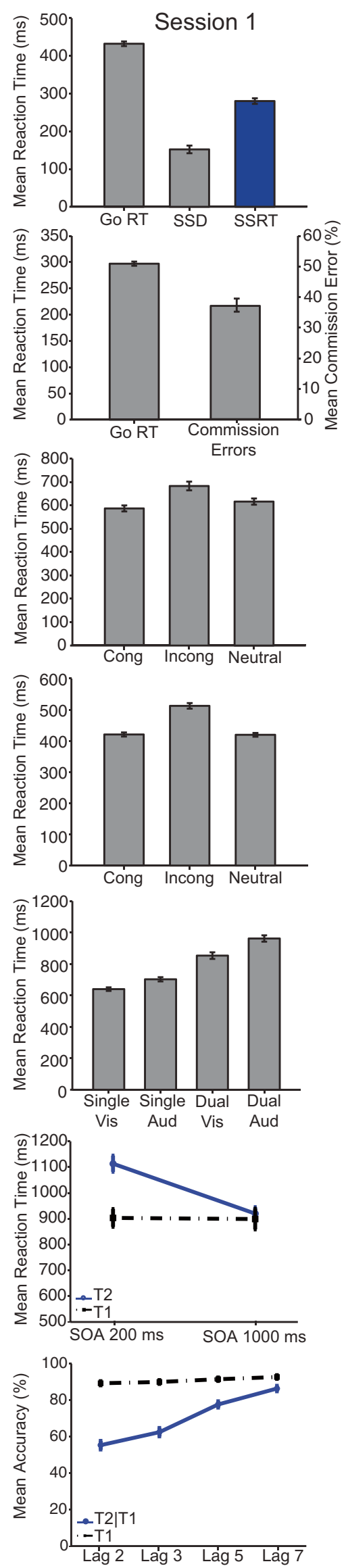
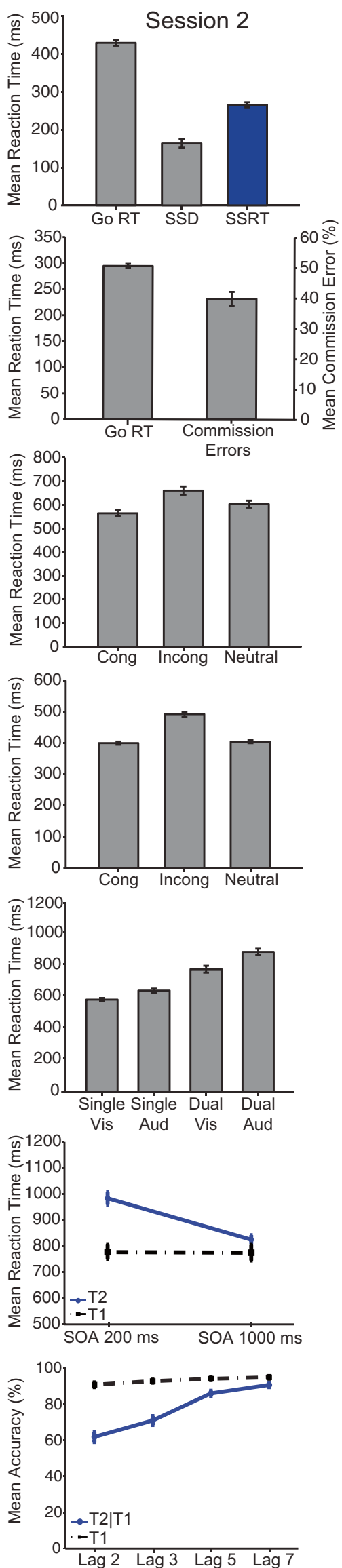
pointed in the same direction as the target arrow (e.g., $\gg>>>$ ). On incongruent trials, the target was flanked by two arrows on each side that pointed in the opposite direction from the target arrow (e.g., $>><>>$ ). On neutral trials, the flankers were two horizontal lines that appeared on each side (e.g., $-<-$ ). The fixation cross was replaced by the concurrent onset of the target and flankers, which remained on the screen for $200 \mathrm{~ms}$. Participants completed 24 practice trials followed by four blocks of 36 test trials. There were equal numbers of trials per condition, and the order of trial types was randomized. The dependent variable was the "flanker congruency effect," which was calculated as the difference in RTs between the congruent and incongruent trials. Lower RT difference scores represented better performance.

\section{Response selection tasks}

Single- vs. dual-response selection task Participants first practiced on three blocks of the two different, twoalternative forced choice (AFC) RT tasks. Block 1 was a visual task in which participants discriminated between two different colored circles (red, RGB 23732 36, and blue, RGB 4471 151; 12 trials), and Block 2 was an auditory discrimination task (two complex tones, previously used in Filmer, Mattingley, Marois, \& Dux, 2013). Each stimulus was mapped to a specific response key and hand ("A" or " $\mathrm{S}$ " for left-hand responses, and " $\mathrm{K}$ " or " $\mathrm{L}$ " for right-hand responses), with the mapping of hand to task counterbalanced across participants. After learning the specific stimulus-response mappings of each task, participants completed a third practice block in which one visual and one auditory stimulus were presented simultaneously on each trial. The experimental phase included four blocks of 36 trials, with each trial type randomly intermixed within blocks. In each test trial, the central fixation cross was followed by a visual stimulus only, an auditory stimulus only, or the simultaneous presentation of both for $200 \mathrm{~ms}$. Participants were instructed to respond as quickly and as accurately as possible via keypress to the stimulus or stimuli. Of key interest was the RT to the single-task trials (averaged across the two tasks), as a pure measure of response selection, and the difference in RTs when the tasks were performed with and without a concurrent task, to measure dual-task executive function. Lower RTs/difference scores indicated better performance.

Psychological refractory period Participants first trained on three blocks of 20 trials of the two different four-AFC tasks. Each task was assigned a response hand and a specific key for each stimulus ("A," "S," "D," or "F" for left-hand responses, and "J," "K," "L," or ";" for right-hand responses), with the mapping of hand to task counterbalanced across participants. In Block 1, participants trained on the visual task (Task 1), which required a manual keypress response to one of four different symbols (\%, \&, @, or \#). Block 2 was an auditory task (Task 2) that required participants to make a manual keypress response to four different complex tones (previously employed in Filmer, Mattingley, Marois, \& Dux, 2013, but different from the tones used in the single- vs. dual-response selection task here). After learning the specific stimulus-response mappings of each task, participants completed a third practice block that was identical to the trials used in the experimental phase. Here, participants were presented with the visual Target 1 (T1) stimulus, which was presented centrally for a variable interval between 200 and $600 \mathrm{~ms}$ (randomly selected at the beginning of each trial). ${ }^{1}$ After either a long $(1,000 \mathrm{~ms})$ or short $(200 \mathrm{~ms})$ stimulus onset asynchrony (SOA), the second, auditory target (T2) was presented for $200 \mathrm{~ms}$. Participants were instructed to respond as quickly and as accurately as possible to the two tasks. The experimental phase consisted of four blocks, each containing 40 trials, with the two SOA conditions being presented equally often across blocks. The dependent variable was the PRP effect, which was calculated by subtracting the T2 RT in the 1,000 ms condition from that in the 200-ms condition (Van Selst, Ruthruff, \& Johnston, 1999). Lower differences scores indicated better performance.

Attentional blink Here, on each trial, participants were presented with a rapid serial visual presentation (RSVP) stream containing two targets (black letters of the alphabet, excluding $\mathrm{I}, \mathrm{L}, \mathrm{O}, \mathrm{Q}, \mathrm{U}, \mathrm{V}$, and $\mathrm{X}$ ) and eight distractors (black digits ranging from 2 to 9). The participants' task was to report the identity of both targets at the end of the stream. There were no time constraints for responding, and participants were encouraged to guess if they were unsure. The fixation cross was replaced by the first stimulus in the RSVP stream. Each item was presented for $100 \mathrm{~ms}$. T1 always appeared at Serial Position 3, and T2 appeared either $200 \mathrm{~ms}$ (lag 2), $300 \mathrm{~ms}$ (lag 3), $500 \mathrm{~ms}$ (lag 5), or $700 \mathrm{~ms}$ (lag 7) after T1. Participants completed 24 practice trials and four test blocks of 24 trials. The dependent variable was $\mathrm{AB}$ magnitude, which was calculated by subtracting the average T2 $\mid \mathrm{T} 1$ accuracy of lags 2 and 3 from lags 5 and 7 (Kelly \& Dux, 2011). A smaller AB magnitude indicated better performance.

\section{Results}

Outlier screening was performed for each participant, in each session and for each task separately. Trials greater than three standard deviations above the mean were removed.

\footnotetext{
${ }^{1}$ Due to a programming error, the presentation duration of the first target varied between 200 and $600 \mathrm{~ms}$ in the PRP paradigm (this was the same value as the fixation duration). The presentation duration of the second target (the critical stimulus) was fixed at 200 or $1,000 \mathrm{~ms}$. Despite this error, the results revealed a robust PRP effect (see Fig. 1).
} 
a

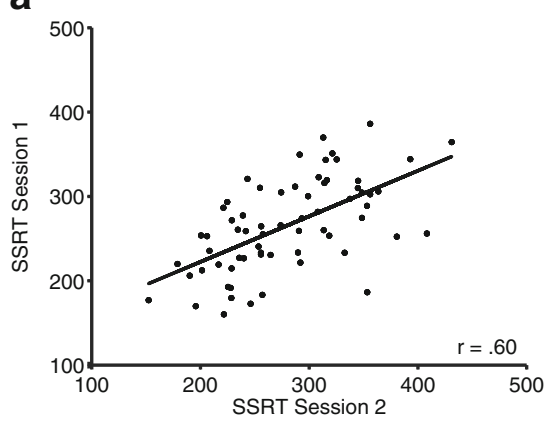

d

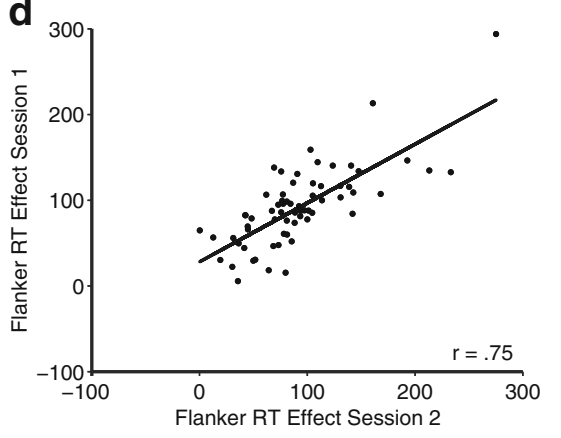

b

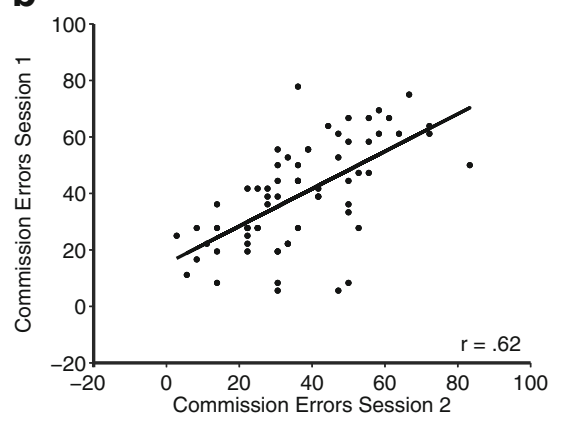

e

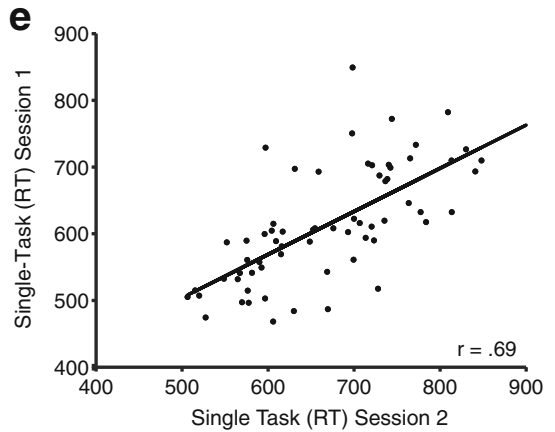

g

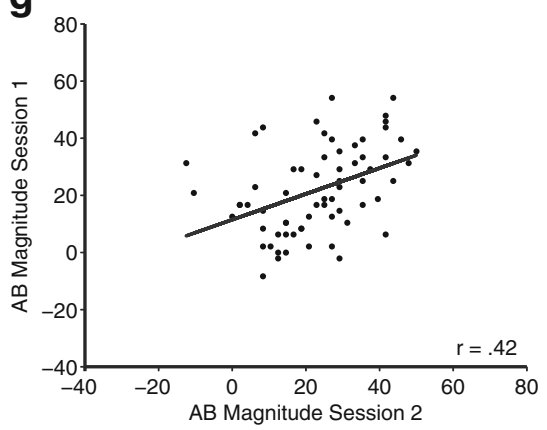

C
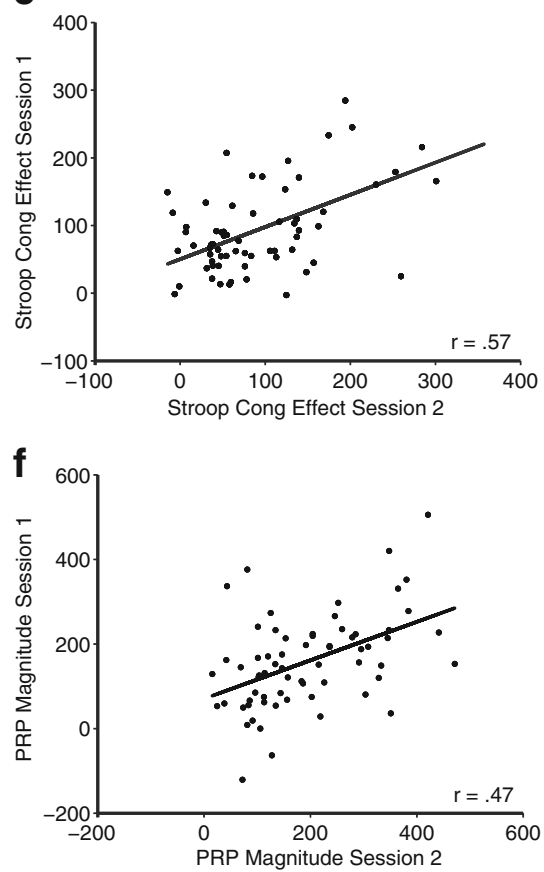

Fig. 2 Scatterplots of the relationship between an individual's (A) stopsignal response time (SSRT), (B) go-no-go commission errors, (C) Stroop congruency effects (Stroop Cong Effect), (D) flanker response time (RT) effect, (E) single-task RTs, (F) psychological refractory period magnitude (PRP Magnitude), and (G) attentional blink magnitudes (AB Magnitude) across Sessions 1 and 2

\section{Test-retest reliability}

To assess the stability of performance for each measure, we first calculated the test-retest reliability between Sessions 1 and 2 for each paradigm. As can be seen in Fig. 2, all seven paradigms were shown to have moderate to strong test-retest reliability $(r$ range $=.42-.69)$, demonstrating that individual task performance remained relatively stable across the seven days. It was therefore appropriate to use these measures in intercorrelation analyses with the other tasks.

\section{Relationships among the tasks}

To assess whether the data contained clusters of interrelated variables that were in line with our a priori predictions, we first explored the relationships between the different tasks. The measures of interest (SST, SSRT; go-no-go, commission errors [\%]; Stroop, congruency effect; flanker, congruency effect $^{2}$; dual- vs. single-response selection task, dual-task cost and single-task response selection RT; PRP, PRP magnitude; $\mathrm{AB}, \mathrm{AB}$ magnitude) were first collapsed across sessions for each paradigm, to obtain an overall score. An attenuation correction $^{3}$ (Hunter \& Schmidt, 1990) was applied to each correlation $\left(r_{\mathrm{c}}\right)$ to control for differences in reliability due to measurement error (see Table 1 and Supplemental Fig. 1). Out of

\footnotetext{
${ }^{2}$ We chose the incongruent-minus-congruent contrast instead of incongruent minus neutral or neutral minus congruent as our main flanker and Stroop interference measure, because this resulted in the largest interference effect.

${ }^{3}$ Attenuation correction formula: $r x y$, corrected $=\operatorname{rxy} / \mathrm{sqrt}(\mathrm{rxx}) * \operatorname{sqrt}(\mathrm{ryy})$ [sqrt $=$ square root], where rxy is the uncorrected correlation taken from the correlation matrix, and rxx and ryy are the test-retest reliability measures for Measures 1 and 2 (Hunter \& Schmidt, 1990).
} 
Table 1 Attenuated Pearson $r$ correlations (Hunter \& Schmidt, 1990) between the stop-signal (SST), go-no-go (No-Go), Stroop, flanker, dual vs. single response selection (Single-Task), psychological refractory period (PRP), and attentional blink ( $\mathrm{AB})$ tasks

\begin{tabular}{|c|c|c|c|c|c|c|c|}
\hline & SST & No-Go & Stroop & Flanker & Single-Task & PRP & $\mathrm{AB}$ \\
\hline 1. SST & - & & & & & & \\
\hline 2. No-Go & $.88^{* *}$ & - & & & & & \\
\hline 3. Stroop & .10 & .21 & - & & & & \\
\hline 4. Flanker & .15 & -.08 & .03 & - & & & \\
\hline 5. Single-Task & -.10 & -.13 & .31 & .14 & - & & \\
\hline 6. PRP & $.38^{*}$ & .25 & $.57^{* * *}$ & -.09 & $.53^{* * *}$ & - & \\
\hline 7. $\mathrm{AB}$ & .30 & .22 & .21 & -.16 & .11 & .36 & - \\
\hline
\end{tabular}

the response selection measures, PRP correlated positively with other measures of response selection, such as the single-response selection $\left(r_{\mathrm{c}}=.53, p<.01\right)$ task, and only marginally with the $\mathrm{AB}\left(r_{\mathrm{c}}=.36, p=.06\right)$ measure. Interestingly, the Stroop task, often employed to measure inhibition-related processes, did not significantly correlate with any of the inhibitory control tasks, but instead correlated significantly positively with the PRP $\left(r_{\mathrm{c}}=.57, p<.01\right)$. The PRP also correlated significantly positively with the SST $\left(r_{\mathrm{c}}=\right.$ $.38, p<.05)$ response inhibition measure. In addition, the SST significantly positively correlated with the go-no-go $\left(r_{\mathrm{c}}=.88\right.$, $p<.01)$ paradigm. Of interest, the flanker paradigm did not correlate with any of the other measures, and was therefore removed from further analysis.

\section{Confirmatory factor analysis}

To examine how response inhibition and response selection processes are related, we performed a series of CFAs. Using the well-established tasks that are known to differ in their response selection and inhibition requirements, we first empirically compared a set of different models (see Table 2) and selected the best-fitting model via multiple appropriate fit indices. The fit of each model was evaluated with the chi-square statistic, the standardized root-mean-square residual (SRMR), and Akaike's information criterion (AIC). The chi-square statistic (nonsignificant values indicating a satisfactory fit) and AIC (lower AIC values indicate better fit) both measure the fit between the observed and predicted covariances. The SRMR represents the square root of the average covariance residuals between the observed and predicted models, with SRMR values less than .08 indicating an acceptable fit, and values less .05 indicating a good fit (Hu \& Bentler, 1998). In addition, we examined the models with Bentler's comparative fit index (CFI), which compares each model to an independent baseline model (i.e., a model in which all covariances are set to zero). Here, values between .95 and 1.00 are indicative of a good model fit (Hu \& Bentler, 1998).

To examine whether one model significantly improved the model fit compared to another, chi-square difference tests were performed on nested, more restrictive models. For these calculations, the chi-square value (as well as the degrees of freedom) of the full model is subtracted from that of the smaller, more restricted model. If the chi-square difference is nonsignificant, then the more restrictive model represents a significantly better fit. The factor models (see Fig. 3) include the standardized factor loadings (standardized regression coefficients), the error variance for each task (including measurement error and idiosyncratic task requirements), and the correlations between the latent variables.

First, we constructed a two-factor model of response selection and response inhibition by selecting the key performance
Table 2 Fit statistics for the confirmatory factor analysis models

\begin{tabular}{lllllll}
\hline Model & $\chi^{2}$ & $d f$ & $p$ & SRMR & CFI & AIC \\
\hline $\begin{array}{l}\text { 1. Two factors: Response Inhibition and } \\
\quad \text { Response Selection (AB incl.) }\end{array}$ & 4.32 & 8 & .83 & .057 & 1.00 & $1,103.92$ \\
$\begin{array}{l}\text { 2. Two factors: Response Inhibition and } \\
\quad \text { Response Selection (AB excl.) }\end{array}$ & 3.33 & 4 & .50 & .057 & 1.00 & 915.68 \\
$\begin{array}{l}\text { 3. One factor: General Action Control } \\
\text { Single-task measure replaced by dual-task cost: }\end{array}$ & 15.75 & 9 & .07 & .106 & .77 & $1,113.35$ \\
$\begin{array}{l}\text { 4. Two factors: Response Inhibition and } \\
\text { Response Selection (AB incl.) }\end{array}$ & 5.87 & 8 & .66 & .071 & 1.00 & $1,106.99$ \\
\hline
\end{tabular}

Chi-square values not significant at the .05 level indicate acceptable fit to the data. Lower standardized root-meansquare residuals (SRMRs) indicate better fit, with SRMR $<.05$ indicating a good fit to the data and SRMR $<.08$ indicating a fair fit to the data. Bentler's comparative fit index (CFI) values $>.95$ indicate excellent fit. Lower values of Akaike's information criterion (AIC) indicate a better-fitting model. ${ }^{*} p<.05$ 
Fig. 3 Best-fitting model (Model 1) of the action control functions. Standardized factor loadings are given next to the straight arrows leading from the latent factors to the indicators. The standardized residual variance for each task is listed beside the error term circle. The number between the two latent factors is the correlation between them. Stop-Signal = stop-signal task; Go-Nogo = gono-go task; PRP = psychological refractory period task; Stroop = Stroop task; Single-Task $=$ single vs. dual-response selection task; $\mathrm{AB}=$ attentional blink task

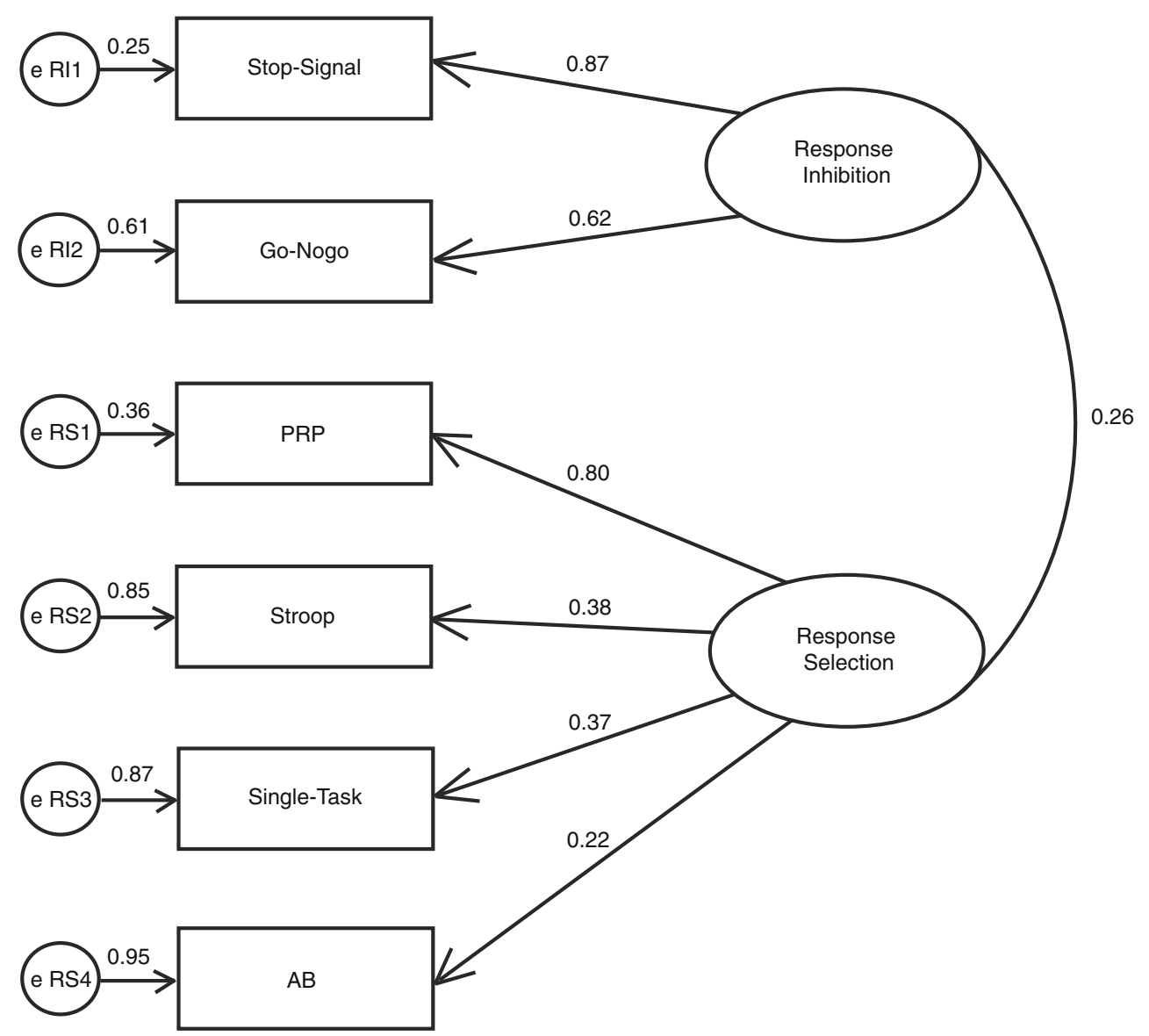

measure for each task as an indicator (e.g., AB represents the AB magnitude). Given that the Stroop task only correlated significantly with response selection measures and not, as anticipated, with any inhibitory control measures, performance on the PRP, Stroop, single-task response selection, and AB served as indicators of the response selection factor. We specifically chose single-task response selection performance instead of dual-task cost as a response selection indicator in order to ensure that the latent response selection variable was not merely driven by dual-task measures (i.e., multitasking). Please note, however, that using the dual-task cost as a response selection indicator still resulted in an acceptable fit (see Table 2, Model 4). The SST and go-no-go task served as indicators of the Response Inhibition factor. The fit between the hypothesized model and the data was acceptable, with a nonsignificant chi-square value $\left[\chi^{2}(8)=4.32, p=.83\right.$; SMRI $=.057, \mathrm{CFI}=1.00$, and an AIC value of 1,103.92). As is shown in Fig. 3, the correlation between response selection and response inhibition was relatively weak $(r=.26)$.

However, because the coefficient predicting performance on the AB from the Response Selection factor was relatively small, we performed post-hoc model modifications to see whether it was possible to develop a better-fitting model. In our second model, we dropped the $\mathrm{AB}$ variable and reestimated the model $\left[\chi^{2}(4)=3.33, p=.5 ; \mathrm{SMRI}=.06, \mathrm{CFI}=1.00\right.$, and an $\mathrm{AIC}$ value of 915.68]. Both the chi-square and the AIC indicated a slightly better-fitting model (see Table 2) after performance, but dropping the $\mathrm{AB}$ as an indicator did not significantly improve the model fit $\left[\Delta \chi^{2}(4)=0.99, p=.91\right]$. Given the nonsignificant improvement in overall fit, the full two-factor model (Model 1) was considered the more parsimonious model.

Next, to test whether response selection and response inhibition processes are reflective of a unitary mechanism, we constructed a general action control one-factor model that collapsed the response selection and response inhibition variables into a single general action control latent variable. As is shown in Table 2, the fit of the one-factor model (Model 3) was poor $\left[\chi^{2}(9)=15.75, p=.07 ; \mathrm{SMRI}=0.106, \mathrm{CFI}=.77\right.$, and an $\mathrm{AIC}$ value of $1,113.35]$. Moreover, it provided a significantly worse fit than the full two-factor model (Model 1), $\Delta \chi^{2}(1)=$ $11.43, p<.001$. Therefore, the full two-factor model, in which tasks were parsed in terms of response selection and inhibition requirements (Model 1), was supported over a single, General Action Control factor model.

\section{Discussion}

We used an individual differences approach to examine the underlying relationship between two cognitive-control 
functions - the selection of appropriate responses (i.e., response selection) and the inhibition of automatic and inappropriate responses (i.e., response inhibition) — at the latent variable level. A series of models were tested via CFA to assess the relations between six behavioral tasks that have been hypothesized to tap response selection and inhibition processes. The results from the CFA showed that the full two-factor model (Model 1), in which tasks were assigned in terms of response selection and response inhibition requirements, provided a significantly better fit to the observed data than did the simplest model - a single General Action Control factor. Specifically, we found that variance on the Response Inhibition factor contributed to performance on the stopsignal and go-no-go tasks, whereas variance on the Response Selection factor played an important role in the PRP, Stroop, and single-task paradigms, and to a smaller degree in the $\mathrm{AB}$.

The present findings suggest that response selection and response inhibition processes are separable, nonunitary processes, since these two factors were weakly related (covariance between the two factors $=.26$ ). Our findings challenge recent work that has suggested that a common mechanism accounts for both the selection and inhibition of actions (Mostofsky \& Simmonds, 2008; Verbruggen et al., 2014). Instead, the dissociation we found reinforces previous CFA studies that have supported the diversity of other executive functions, as well as some overlap (Fisk \& Sharp, 2004; Friedman \& Miyake, 2004; Miyake et al., 2000). Moreover, the present work fits well with behavioral evidence from Yamaguchi et al. (2012), who found that the decision to stop an inappropriate response is not dependent on the processing capacity at the central response-selection stage. In addition, our results are consistent with neuroimaging and neurostimulation findings that have posited that distinct neural substrates are involved in response selection (Dux et al., 2006; Filmer, Mattingley, Marois, \& Dux, 2013) and response inhibition processes (Aron et al., 2014).

Interestingly, we did not find that performance on the flanker task correlated significantly with the Stroop task or any of the other performance measures. Although there is evidence of commonly observed activations in the anterior cingulate cortex and prefrontal cortex when dealing with response conflict in the flanker and Stroop tasks (Fan, Flombaum, McCandliss, Thomas, \& Posner, 2003), studies adopting an individual differences paradigm have frequently shown that correlations vary between the two tasks and other cognitive measures such as intelligence (Jensen \& Rohwer, 1966; Spilsbury, 1992; Stins, Polderman, Boomsma, \& de Geus, 2005). Moreover, factorially combined Stroop and Simon tasks and flanker and Simon tasks have shown that the conflict-elicited control mechanisms for the two tasks seem to operate in a conflict-specific (i.e., context-specific conflict triggers particular cognitive-control processes), rather than a conflict-general manner that would act on any type of task conflict (for a review, see Egner, 2008). These findings therefore suggest that these types of conflict tasks may have different cognitive-control functions that are mediated by independent cognitive-control loops.

The finding that performance on the Stroop task is more dependent on the constraints imposed by response selection, rather than the ability to inhibit distracting automatic responses, contrasts with existing studies that have utilized CFA methods. In particular, Miyake and colleagues found that the Stroop task significantly loaded on the prepotent Response Inhibition factor (Friedman \& Miyake, 2004; Miyake et al., 2000). Instead, our finding more closely resembles previous research that has found that incongruent trials (e.g., the word "blue" presented in red) create interference at the response selection level (Cohen, Dunbar, \& McClelland, 1990; MacLeod, 1991; MacLeod \& MacDonald, 2000; Stafford \& Gurney, 2007), such that pressing a key associated with a specific color (e.g., red) competes with the color word (e.g., the word "blue") as a potential alternative response. Thus, despite the fact that the Stroop task is typically considered to reflect response inhibition, the combination of these prior findings and ours instead implies that the Stroop paradigm actually taps response selection processes similar to those elicited by the PRP and single-response selection task paradigms.

It must be noted that the $\mathrm{AB}$ only weakly loaded on the Response Selection factor. Because this protocol was the only paradigm out of the six submitted to CFA that contained distractor items, it may be possible that this paradigm predominantly measures the suppression of distracting and irrelevant stimuli through controlled attention, which may be separate from the controlled inhibition of prepotent motor responses that is required in the stop-signal and go-no-go tasks (Friedman \& Miyake, 2004; Nigg, 2000). The weak correlation between the $\mathrm{AB}, \mathrm{PRP}$, and single-response selection tasks also suggests that performance in the $\mathrm{AB}$ may predominantly rely on another mechanism dissociated from response selection. A similar finding has been noted in two recent studies by Garner and colleagues (Garner, Matthews, Remington, \& Dux, 2015; Garner, Tombu, \& Dux, 2014), who investigated whether response selection and sensory consolidation rely on the same capacitylimited central mechanism (Jolicœur \& Dell'Acqua, 1998). For example, in the Garner et al. (2014) study, participants were randomly assigned to a relevant training group (a speeded forced choice sensory-motor task matching $\mathrm{T} 1$ for an $\mathrm{AB}$ or PRP task), an irrelevant training group (i.e., a speeded forced choice sensory-motor task not matching T1 for an AB or PRP task), or a control group (no training). The authors showed that although only relevant training attenuated the PRP effect, both relevant and irrelevant training reduced the $\mathrm{AB}$, suggesting that $\mathrm{AB}$ performance is at least partly driven by a different underlying mechanism (but see Jolicœur, 1998; Tombu et al., 2011, for evidence of overlap). 
While we argue for a response selection-inhibition account of the observed two-factor solution, one alternate interpretation is that these components could reflect alternative cognitive processes, such as attentional monitoring or working memory. Specifically, performance in response inhibition tasks is often thought to rely heavily on attentional monitoring when one is preparing to respond to infrequent stimuli or conflict (Duann, Ide, Luo, \& Li, 2009; Erika-Florence, Leech, \& Hampshire, 2014; Hampshire, Chamberlain, Monti, Duncan, \& Owen, 2010), whereas performance in response selection paradigms may be dependent on working memory maintenance processes that support reasoning and rule-processing demands (Bunge, Hazeltine, Scanlon, Rosen, \& Gabrieli, 2002; Jiang \& Kanwisher, 2003; Miller \& Cohen, 2001). If our two-factor solution did instead reflect attentional monitoring and working memory processes, then one would predict that the conflict monitoring generated by an incongruent flanker stimuli (the flanker task) and the working memory requirements needed for the sensory consolidation of Targets 1 and 2 (the AB task) should also correlate significantly with one of these two factors. Because the flanker paradigm was omitted from our CFA analysis due to its very weak zero-order correlations with the remaining six paradigms, and because the AB only weakly loaded on the Response Selection factor, our findings instead favor the response selection-inhibition account.

In summary, the present findings support the hypothesis that response selection and response inhibition tap two distinct mechanisms of action control. These results reconcile the previous conflicting empirical work into the nature of response selection and inhibition processes by using an individual differences latent variable approach and a range of cognitive tasks to identify the common sources of variance associated with each process. The results have important implications for how we should conceptualize action control and how one might tailor interventions designed to overcome the limitations associated with each domain. In other words, our findings illustrate the value of using multiple response selection and response inhibition paradigms when investigating these two cognitive operations, and they further suggest that interventions need to be specialized for each aspect of action control.

\footnotetext{
Author note A.D.B., H.L.F., K.G.G., and P.E.D. designed the experiment. All tasks were programmed by C.K.N. and A.D.B., with the analysis script written by C.K.N. The testing and data collection were performed by A.D.B., who also performed the data analysis and interpretation under the supervision of H.L.F. and P.E.D. A.D.B. drafted the manuscript, and H.L.F., K.G.G., C.K.N., and P.E.D. provided critical revisions. All authors approved the final version of the manuscript for submission. The authors declare that they have no conflicts of interest with respect to their authorship or the publication of this article. This work was supported by the ARC-SRI Science of Learning Research Centre (Grant No. SR120300015 to P.E.D.), an Australian Postgraduate Award to A.D.B., and an ARC Future Fellowship to P.E.D. (No. FT120100033).
}

\section{References}

Aron, A. R., Fletcher, P. C., Bullmore, E. T., Sahakian, B. J., \& Robbins, T. W. (2003). Stop-signal inhibition disrupted by damage to right inferior frontal gyrus in humans. Nature Neuroscience, 6, 115-116. doi: $10.1038 / \mathrm{nn} 1003$

Aron, A. R., Robbins, T. W., \& Poldrack, R. A. (2014). Inhibition and the right inferior frontal cortex: One decade on. Trends in Cognitive Sciences, 18, 177-185. doi:10.1016/j.tics.2013.12.003

Bentler, P. M., \& Chou, C.-P. (1987). Practical issues in structural modeling. Sociological Methods \& Research, 16, 78-117. doi:10.1177/ 0049124187016001004

Brainard, D. H. (1997). The Psychophysics Toolbox. Spatial Vision, 10, 433-436. doi:10.1163/156856897X00357

Brown, S. D., \& Heathcote, A. (2008). The simplest complete model of choice response time: Linear ballistic accumulation. Cognitive Psychology, 57, 153-178. doi:10.1016/j.cogpsych.2007.12.002

Buch, E. R., Mars, R. B., Boorman, E. D., \& Rushworth, M. F. (2010). A network centered on ventral premotor cortex exerts both facilitatory and inhibitory control over primary motor cortex during action reprogramming. Journal of Neuroscience, 30, 1395-1401. doi:10. 1523/JNEUROSCI.4882-09.2010

Bunge, S. A. (2004). How we use rules to select actions: A review of evidence from cognitive neuroscience. Cognitive, Affective, \& Behavioral Neuroscience, 4, 564-579. doi:10.3758/CABN.4.4.564

Bunge, S. A., Hazeltine, E., Scanlon, M. D., Rosen, A. C., \& Gabrieli, J. D. E. (2002). Dissociable contributions of prefrontal and parietal cortices to response selection. NeuroImage, 17, 1562-1571.

Chambers, C. D., Bellgrove, M. A., Gould, I. C., English, T., Garavan, H., McNaught, E., .. . Mattingley, J. B. (2007). Dissociable mechanisms of cognitive control in prefrontal and premotor cortex. Journal of Neurophysiology, 98, 3638-3647. doi:10.1152/jn.00685.2007

Chambers, C. D., Bellgrove, M. A., Stokes, M. G., Henderson, T. R., Garavan, H., Robertson, I. H., . . . Mattingley, J. B. (2006). Executive "brake failure" following deactivation of human frontal lobe. Journal of Cognitive Neuroscience, 18, 444 455. doi:10.1162/ 089892906775990606

Cohen, J. D., Dunbar, K., \& McClelland, J. L. (1990). On the control of automatic processes: A parallel distributed processing account of the Stroop effect. Psychological Review, 97, 332-361. doi:10.1037/ 0033-295X.97.3.332

Crews, F. T., \& Boettiger, C. A. (2009). Impulsivity, frontal lobes and risk for addiction. Pharmacology Biochemistry and Behavior, 93, 237 247. doi:10.1016/j.pbb.2009.04.018

Donders, F. C. (1969). On the speed of mental processes. In W. G. Koster (Ed.), Attention and performance II (pp. 412-431). Amsterdam, The Netherlands: North-Holland. doi:10.1016/0001-6918(69) 90065-1

Duann, J. R., Ide, J. S., Luo, X., \& Li, C. S. (2009). Functional connectivity delineates distinct roles of the inferior frontal cortex and presupplementary motor area in stop signal inhibition. Journal of Neuroscience, 29, 10171-10179. doi:10.1523/JNEUROSCI.130009.2009

Duncan, J., \& Owen, A. M. (2000). Common regions of the human frontal lobe recruited by diverse cognitive demands. Trends in Neurosciences, 23, 475-483.

Dux, P. E., \& Harris, I. M. (2007). On the failure of distractor inhibition in the attentional blink. Psychonomic Bulletin \& Review, 14, 723-728. doi:10.3758/BF03196828

Dux, P. E., Ivanoff, J., Asplund, C. L., \& Marois, R. (2006). Isolation of a central bottleneck of information processing with time-resolved FMRI. Neuron, 52, 1109-1120. doi:10.1016/j.neuron.2006.11.009

Dux, P. E., \& Marois, R. (2008). Distractor inhibition predicts individual differences in the attentional blink. PLOS ONE, 3, e3330. doi:10. 1371/journal.pone. 0003330 
Dux, P. E., \& Marois, R. (2009). The attentional blink: A review of data and theory. Attention, Perception, \& Psychophysics, 71, 1683-1700. doi:10.3758/APP.71.8.1683

Dux, P. E., Tombu, M. N., Harrison, S., Rogers, B. P., Tong, F., \& Marois, R. (2009). Training improves multitasking performance by increasing the speed of information processing in human prefrontal cortex. Neuron, 63, 127-138. doi:10.1016/j.neuron.2009.06.005

Egner, T. (2008). Multiple conflict-driven control mechanisms in the human brain. Trends in Cognitive Sciences, 12, 374-380. doi:10. 1016/j.tics.2008.07.001

Erika-Florence, M., Leech, R., \& Hampshire, A. (2014). A functional network perspective on response inhibition and attentional control. Nature Communication, 5, 4073. doi:10.1038/ncomms5073

Eriksen, C. W., \& Schultz, D. W. (1979). Information processing in visual search: A continuous flow conception and experimental results. Perception \& Psychophysics, 25, 249-263. doi:10.3758/ BF03198804

Fan, J., Flombaum, J. I., McCandliss, B. D., Thomas, K. M., \& Posner, M. I. (2003). Cognitive and brain consequences of conflict. NeuroImage, 18, 42-57. doi:10.1006/nimg.2002.1319

Filmer, H. L., Mattingley, J. B., Marois, R., \& Dux, P. E. (2013). Disrupting prefrontal cortex prevents performance gains from sensory-motor training. Journal of Neuroscience, 33, 18654 18660. doi:10.1523/JNEUROSCI.2019-13.2013

Filmer, H. L., Mattingley, J. B., \& Dux, P. E. (2013). Improved multitasking following prefrontal tDCS. Cortex, 49, 2845-2852. doi:10. 1016/j.cortex.2013.08.015

Fisk, J. E., \& Sharp, C. A. (2004). Age-related impairment in executive functioning: Updating, inhibition, shifting, and access. Journal of Clinical and Experimental Neuropsychology, 26, 874-890. doi:10. 1080/13803390490510680

Friedman, N. P., \& Miyake, A. (2004). The relationship among inhibition and interference control functions: A latent-variable analysis. Journal of Experimental Psychology: General, 133, 101-135. doi: 10.1037/0096-3445.133.1.101

Garner, K. G., Matthews, N., Remington, R. W., \& Dux, P. E. (2015). Transferability of training benefits differs across neural events: Evidence from ERPs. Journal of Cognitive Neuroscience, 27, 2079-2094. doi:10.1162/jocn_a 00833

Garner, K. G., Tombu, M. N., \& Dux, P. E. (2014). The influence of training on the attentional blink and psychological refractory period. Attention, Perception, \& Psychophysics, 76, 979-999. doi:10.3758/ s13414-014-0638-y

Hampshire, A., Chamberlain, S. R., Monti, M. M., Duncan, J., \& Owen, A. M. (2010). The role of the right inferior frontal gyrus: Inhibition and attentional control. Neurolmage, 50, 1313-1319. doi:10.1016/j. neuroimage.2009.12.109

Harnishfeger, K. K. (1995). The development of cognitive inhibition: Theories, definitions, and research evidence. In F. N. D. C. J. Brainerd (Ed.), Interference and inhibition in cognition (pp. 175204). San Diego, CA: Academic Press.

Houben, K. (2011). Overcoming the urge to splurge: Influencing eating behavior by manipulating inhibitory control. Journal of Behavior Therapy and Experimental Psychiatry, 42, 384-388. doi:10.1016/j. jbtep.2011.02.008

Hu, L., \& Bentler, P. M. (1998). Fit indices in covariance structure modeling: Sensitivity to underparameterized model misspecification. Psychological Methods, 3, 424-453.

Hunter, J. E., \& Schmidt, F. L. (1990). Methods of meta-analysis: Correcting error and bias in research findings. Beverly Hills, CA: Sage.

Jensen, A. R., \& Rohwer, W. D., Jr. (1966). The Stroop color-word test: A review. Acta Psychologica, 25, 36-93.

Jiang, Y., \& Kanwisher, N. (2003). Common neural mechanisms for response selection and perceptual processing. Journal of Cognitive Neuroscience, 15, 1095-1110. doi:10.1162/089892903322598076
Jolicœur, P. (1998). Modulation of the attentional blink by on-line response selection: Evidence from speeded and unspeeded task1 decisions. Memory \& Cognition, 26, 1014-1032. doi:10.3758/ BF03201180

Jolicœur, P., \& Dell'Acqua, R. (1998). The demonstration of short-term consolidation. Cognitive Psychology, 36, 138-202. doi:10.1006/ cogp.1998.0684

Kelly, A. J., \& Dux, P. E. (2011). Different attentional blink tasks reflect distinct information processing limitations: An individual differences approach. Journal of Experimental Psychology: Human Perception and Performance, 37, 1867-1873. doi:10.1037/ a0025975

Lappin, J. S., \& Eriksen, C. W. (1966). Use of a delayed signal to stop a visual reaction-time response. Journal of Experimental Psychology, 72, 805-811. doi:10.1037/h0021266

Logan, G. D., \& Burkell, J. (1986). Dependence and independence in responding to double stimulation: A comparison of stop, change, and dual-task paradigms. Journal of Experimental Psychology: Human Perception and Performance, 12(4), 549-563. doi:10. 1037/0096-1523.12.4.549

Logan, G. D., \& Cowan, W. B. (1984). On the ability to inhibit thought and action: A theory of an act of control. Psychological Review, 91, 295-327. doi:10.1037/0033-295X.91.3.295

MacLeod, C. M. (1991). Half a century of research on the Stroop effect: An integrative review. Psychological Bulletin, 109, 163-203. doi: 10.1037/0033-2909.109.2.163

MacLeod, C. M., \& MacDonald, P. A. (2000). Interdimensional interference in the Stroop effect: Uncovering the cognitive and neural anatomy of attention. Trends in Cognitive Sciences, 4, 383-391. doi:10. 1016/S1364-6613(00)01530-8

Miller, E. K., \& Cohen, J. D. (2001). An integrative theory of prefrontal cortex function. Annual Review of Neuroscience, 24, 167-202. doi: 10.1146/annurev.neuro.24.1.167

Miyake, A., Friedman, N. P., Emerson, M. J., Witzki, A. H., Howerter, A., $\&$ Wager, T. D. (2000). The unity and diversity of executive functions and their contributions to complex "frontal lobe" tasks: A latent variable analysis. Cognitive Psychology, 41, 49-100. doi:10. 1006/cogp.1999.0734

Mostofsky, S. H., \& Simmonds, D. J. (2008). Response inhibition and response selection: Two sides of the same coin. Journal of Cognitive Neuroscience, 20, 751-761. doi:10.1162/jocn.2008.20500

Nigg, J. T. (2000). On inhibition/disinhibition in developmental psychopathology: Views from cognitive and personality psychology and a working inhibition taxonomy. Psychological Bulletin, 126, 220246. doi:10.1037/0033-2909.126.2.220

Nigg, J. T. (2001). Is ADHD a disinhibitory disorder? Psychological Bulletin, 127, 571-598. doi:10.1037/0033-2909.127.5.571

Noel, X., Brevers, D., \& Bechara, A. (2013). A neurocognitive approach to understanding the neurobiology of addiction. Current Opinion in Neurobiology, 23, 632-638. doi:10.1016/j.conb.2013.01.018

Norman, D., \& Shallice, T. (1986). Attention to action: Willed and automatic control of behaviour. In R. G. S. R. Davidson \& D. Shapiro (Eds.), Consciousness and self-regulation: Advances in research and theory (pp. 1-18). New York, NY: Plenum Press.

Pashler, H. (1984). Processing stages in overlapping tasks: Evidence for a central bottleneck. Journal of Experimental Psychology: Human Perception and Performance, 10, 358-377. doi:10.1037/00961523.10.3.358

Pelli, D. G. (1997). The VideoToolbox software for visual psychophysics: Transforming numbers into movies. Spatial Vision, 10, 437-442. doi:10.1163/156856897X00366

Penades, R., Catalan, R., Rubia, K., Andres, S., Salamero, M., \& Gasto, C. (2007). Impaired response inhibition in obsessive compulsive disorder. European Psychiatry, 22, 404-410. doi:10.1016/j.eurpsy. 2006.05.001 
Ratcliff, R., \& Smith, P. L. (2004). A comparison of sequential sampling models for two-choice reaction time. Psychological Review, 111, 333-367. doi:10.1037/0033-295X.111.2.333

Raymond, J. E., Shapiro, K. L., \& Arnell, K. M. (1992). Temporary suppression of visual processing in an RSVP task: An attentional blink? Journal of Experimental Psychology: Human Perception and Performance, 18, 849-860. doi:10.1037/0096-1523.18.3.849

Schumacher, E. H., Seymour, T. L., Glass, J. M., Fencsik, D. E., Lauber, E. J., Kieras, D. E., \& Meyer, D. E. (2001). Virtually perfect time sharing in dual-task performance: Uncorking the central cognitive bottleneck. Psychological Science, 12, 101-108.

Sigman, M., \& Dehaene, S. (2008). Brain mechanisms of serial and parallel processing during dual-task performance. Journal of Neuroscience, 28, 7585-7598. doi:10.1523/JNEUROSCI.0948-08. 2008

Smith, P. L., \& Ratcliff, R. (2004). Psychology and neurobiology of simple decisions. Trends in Neurosciences, 27, 161-168. doi:10. 1016/j.tins.2004.01.006

Spilsbury, G. (1992). Complexity as a reflection of the dimensionality of a task. Intelligence, 16, 31-45. doi:10.1016/0160-2896(92)90024-L

Stafford, T., \& Gurney, K. N. (2007). Biologically constrained action selection improves cognitive control in a model of the Stroop task. Philosophical Transactions of the Royal Society B, 362, 1671-1684. doi:10.1098/rstb.2007.2060

Stins, J. F., Polderman, J. C., Boomsma, D. I., \& de Geus, E. J. C. (2005). Response interference and working memory in 12-year-old children. Child Neuropsychology, 11, 191-201. doi:10.1080/092970490911351

Stroop, J. R. (1935). Studies of interference in serial verbal reactions (PhD Dissertation). George Peabody College for Teachers, Nashville, TN.

Tombu, M. N., Asplund, C. L., Dux, P. E., Godwin, D., Martin, J. W., \& Marois, R. (2011). A Unified attentional bottleneck in the human brain. Proceedings of the National Academy of Sciences, 108, 13426-13431. doi:10.1073/pnas.1103583108

Usher, M., \& McClelland, J. L. (2001). The time course of perceptual choice: The leaky, competing accumulator model. Psychological Review, 108, 550-592. doi:10.1037/0033-295X.111.3.757 van Gaal, S., Ridderinkhof, K. R., Fahrenfort, J. J., Scholte, H. S., \& Lamme, V. A. F. (2008). Frontal cortex mediates unconsciously triggered inhibitory control. Journal of Neuroscience, 28, 80538062. doi:10.1523/JNEUROSCI.1278-08.2008

Van Selst, M., Ruthruff, E., \& Johnston, J. C. (1999). Can practice eliminate the Psychological Refractory Period effect? Journal of Experimental Psychology: Human Perception and Performance, 25, 1268-1283. doi:10.1037/0096-1523.25.5.1268

Verbruggen, F., Aron, A. R., Stevens, M. A., \& Chambers, C. D. (2010). Theta burst stimulation dissociates attention and action updating in human inferior frontal cortex. Proceedings of the National Academy of Sciences, 107, 13966-13971. doi:10.1073/pnas.1001957107

Verbruggen, F., \& Logan, G. D. (2008). Automatic and controlled response inhibition: Associative learning in the go/no-go and stopsignal paradigms. Journal of Experimental Psychology: General, 137, 649-672. doi:10.1037/a0013170

Verbruggen, F., \& Logan, G. D. (2009). Models of response inhibition in the stop-signal and stop-change paradigms. Neuroscience \& Biobehavioral Reviews, 33, 647-661. doi:10.1016/j.neubiorev. 2008.08.014

Verbruggen, F., \& Logan, G. D. (2015). Evidence for capacity sharing when stopping. Cognition, 142, 81-95. doi:10.1016/j.cognition. 2015.05.014

Verbruggen, F., McLaren, I. P., \& Chambers, C. D. (2014). Banishing the control homunculi in studies of action control and behavior change. Perspectives on Psychological Science, 9, 497-524. doi:10.1177/ 1745691614526414

Welford, A. T. (1952). The "psychological refractory period" and the timing of high-speed performance - A review and a theory. British Journal of Psychology, 43, 2-19.

Wyble, B., Bowman, H., \& Nieuwenstein, M. (2009). The attentional blink provides episodic distinctiveness: Sparing at a cost. Journal of Experimental Psychology: Human Perception and Performance, 35, 787-807. doi:10.1037/a0013902

Yamaguchi, M., Logan, G. D., \& Bissett, P. G. (2012). Stopping while going! Response inhibition does not suffer dual-task interference. Journal of Experimental Psychology: Human Perception and Performance, 38, 123-134. doi:10.1037/a0023918 